\section{MS19 P11}

Hydrogen bonded rings as supramolecular motifs Catharine Esterhuysen, Martin W. Bredenkamp, Leonard J. Barbour Dept. of Chemistry and Polymer Science, University of Stellenbosch, Private Bag X1, Matieland, 7602, SouthAfrica. E-mail: ce@sun.ac.za

Keywords: hydrogen bonding, computational analysis of crystallographic data, DFT

Four and six-membered hydrogen bonded rings are common motifs in supramolecular architectures. Their influence on the formation of certain clathrate structures has been investigated and is described herein. This study includes a computational part at the B3LYP/6-31G(d) level of theory, where the hydrogen bond stabilisation involved when such a hydrogen bonded ring is formed is analysed, and an examination of the Cambridge Structural Database (CSD) [1]. In particular, the presence of the sixmembered hydrogen bonded ring in the solid state structure of racemic Dianin's compound (4-phydroxyphenyl-2,2,4-trimethylchroman) [2] appears to play the most crucial role in the crystallisation of the clathrate, with the formation of the large clathrate void $\left(240 \AA^{3}\right)$. The hydrogen bond stabilisation for a series of model compounds containing four and six-membered hydrogen bonded rings is shown to be substantially greater than that of four or six equivalent individual hydrogen bonds. The effect of cooperativity of hydrogen bonds is further explored.

[1] Allen, F. H. Acta Cryst. 2002, B58, 380.

[2] Dianin, A. P. J. Russ. Phys. Chem. Soc. 1914, 31, 1310.

\section{MS19 P12}

Crystal Structures and Interactions in Packing of Novel Thiadiazolyl - and Triazolyl-benzimidazole Derivatives F. Betül Kaynak ${ }^{a}$, Süheyla Özbey ${ }^{\mathrm{a}}$, Gülgün Ayhan Kılcıgil ${ }^{\mathrm{b}}$, Canan Kuş ${ }^{\mathrm{b}}{ }^{\mathrm{a}}$ Department of Physics Engineering, Hacettepe University, 06800 Beytepe, Ankara, Turkey. ${ }^{\mathrm{b} D e p a r m e n t}$ of Pharmaceutical Chemistry, Pharmacy Phaculty, Ankara University, 06100 Tandoğan, Ankara, Turkey.

E-mail: gulsen@hacettepe.edu.tr

\section{Keywords: pharmaceutical crystallography, crystallography of biological small molecules, crystal packing}

The development of resistance to current antibacterial therapy

continues to derive the search for more effective agents. In addition, primary and opportunistic fungal infections continue to increase the number of immunocompromised patients, those suffering from such as AIDS or cancer or who have undergone organ transplantation. It is well known that benzimidazoles exhibit antimicrobial [1], [2], antitubercular, anticancer, anthelmintic, antiallergic, antioxidant [3], anticonvulsant [4] and analgesic activities. It is also well known that thiadiazoles possess antiinflamatory [5] and antimicrobial [6,7] activities.

In this paper our attention was directed toward the examination of crystal structures, hydrogen bonding characteristics and crystal packings of thiadiazolyl- and triazolyl-benzimidazole derivatives. The crystal and molecular structures of some novel benzimidazole derivatives were determined by single crystal X-ray diffracrion. In the crystal structures there are intra- and intermolecular hydrogen bonds. The crystal packing of all compounds are stabilized by hydrogen bonding and $\mathrm{N}-$ $\mathrm{H} \cdots \mathrm{F} / \mathrm{Cl}, \mathrm{C}-\mathrm{H} \cdots \mathrm{N}$ and $\mathrm{C}-\mathrm{H} \cdots \pi$ interactions.
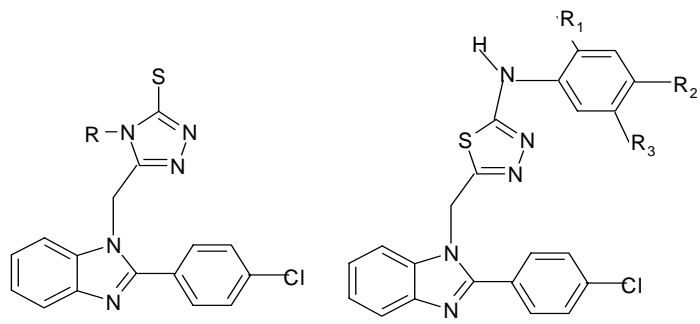

$\mathrm{R}=\mathrm{CH}_{3}, \mathrm{C}_{6} \mathrm{H}_{4} \mathrm{OCH}_{3} ; \mathrm{R}_{1}=\mathrm{F}, \mathrm{H} ; \mathrm{R}_{2}=\mathrm{CH}_{3}, \mathrm{H} ; \mathrm{R}_{3}=\mathrm{Cl}, \mathrm{H}$

[1] Göker, H., Tunçcbilek, M., Ayhan, G. and Altanlar, N. Farmaco, 1998, 53, 415.

[2]Kılcıgil, G.A., Tunçcbilek, M., Altanlar, N. and Göker, H. Farmaco, 1999, 54, 562.

[3] Can-Eke, B., Puskullu, M.O., Buyukbingol, E., Iscan, M., Chemico-Biological Interactions, 1998, 113, 65.

[4] Demirayak, S., Abu Mohsen, U. and Karaburun, A.C., Eur. J. Med. Chem., 2002, 37, 255.

[5] Boschelli, D.H., Connor, D.T., Bornemeier, D.A., Dyer, R.D., Kennedy, J.A., Kuipers, P.J., Okonkwo, G.C., Schrier, D.J., Wright, C.D., Arzeim.-Forsch./Drug Res., 2001, 51,478.

[6]Tsonitis, A., Vasrvaresou, A., Calogeropoulou, T., SiatraPapastaikoudi, T., Tiligada, A., Arzeim.-Forsch./Drug Res., 1997, 47, 307.

[7] Ayhan-Kılcıgil, G., Kuş, C., Altanlar, N., Özbey, S., Turk. J.Chem., 2005, 29, 153 .

\section{MS19 P13}

3-(methoxyanilino)phthalides Mustafa Odabasoğlu ${ }^{\mathrm{a}}$ and Orhan Büyükgüngör ${ }^{b}$, ${ }^{a}$ Department of Chemistry, Ondokuz Mayis University, TR-55139, Samsun, Turkey, ${ }^{\mathrm{b}}$ Department of Physics, Ondokuz Mayis University, TR55139, Samsun, Turkey. E-mail: muodabas@omu.edu.tr

\section{Keywords: phthalide, isobenzofuranon, benzolacton}

Benzolactones (phthalides) are found in plants and are known to show diverse biological activities as hormones, pheromones and antibiotics. These compounds possess several important properties, such as fungicidal, ctericidal and herbicidal, analgesic and hypotensive and vasorelaxant activities. In addition, phthalidederivatives are useful in the treatment of circulatory and heart-related diseases. They are also found to be associated with pesticidal activities. In addition, phthalide is a versatile synthetic building block, particularly for the synthesis of carbocyclic and heterocyclic compounds. We decided to investigate the solid-state structures of 3-substituted phthalides by X-ray diffraction methods and present here the3-(methoxyanilino)phthalides of the title compounds (IIII).

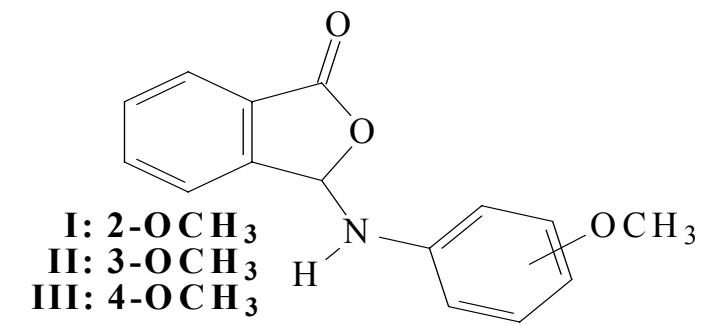


Two enantiomeric molecules of (I) are linked by N-H...O hydrogen bonds. These N-H...O hydrogen bonds generate $\mathrm{R}_{2}{ }^{2}(12)$ rings, which also have two edge-fused $S(5)$ rings, resulting in an $\mathrm{S}(5)\left[\mathrm{R}_{2}{ }^{2}(12)\right] \mathrm{S}(5)$ motif [1]. The structure (II) is stabilized by $\mathrm{N}-\mathrm{H} . . . \mathrm{O}, \mathrm{C}-\mathrm{H} . . \mathrm{O}$ and $\mathrm{C}-\mathrm{H} \ldots \pi$ interactions. The $\mathrm{N}-\mathrm{H} . . . \mathrm{O}$ hydrogen bonds generate two $\mathrm{C}(6)$ chains one within the other and these chains are linked by $\mathrm{C}-\mathrm{H}$... O hydrogen bonds generating and $\mathrm{R}_{2}{ }^{2}(21)$ ring motif. Two inversion-related molecules of (III) are linked by $\mathrm{N}-\mathrm{H}$... O hydrogen bonds generating an $\mathrm{R}_{2}{ }^{2}(12)$ ring motif. There are no direction specific aromatic $\pi-\pi$ interactions between adjacent rings in of (III), but two CH... $\pi$. interactions link parallel phthalide rings [2]. The phthalide part of the molecules are planar in all three compounds and the dihedral angles between the phthalide group and the benzene ring are $63.26(8)^{\circ}, 62.81(8)^{\circ}$ and $71.18(5)^{\circ}$ for I, II and III, respectively.

[1] Odabaş̧oğlu M. \& Büyükgüngör O., Acta Cryst., 2006, E62, o1884-o1885.

[2] Odabaşoğlu M. \& Büyükgüngör O., Acta Cryst., 2006, E62, o1882-o1883.

\section{MS19 P14}

First atomic structure of bacterial S-layer protein reveals a novel protein architecture Tea Pavkov ${ }^{\mathrm{a}}$, Eva M. Egelseer ${ }^{\mathrm{b},}$ Margit Sara $^{\mathrm{b} \dagger}$, Walter Keller $^{\mathrm{a}},{ }^{\mathrm{a}} K . F$. University Graz, Austria. ${ }^{\mathrm{b}}$ Center for Nanobiotechnology, University of Natural Resources and Applied Life Sciences, Vienna, Austria. ${ }^{\dagger}$ deceased May 2006

E-mail: tea.pavkov@uni-graz.at

\section{Keywords: 3D-structure, bacterial S-layer, SAXS}

Surface layer (S-layer) proteins are one of the most abundant cellular proteins with the ability to form a very uniform surface on the prokaryotic cell. Different functions, from protection to recognition, are proposed. In spite of their biological importance for the functionality of prokaryotic cells, high resolution structural information of S-layer proteins is very scarce. The main reason for the absence of 3-D structural information resides in the tendency of S-layer proteins to self-assemble into 2-D crystalline lattices, thereby preventing the formation of 3D crystals .

For obtaining 3D-crystals and determining the structurefunction relationship of $\mathrm{SbsC}$, the S-layer protein from $G$. stearothermophilus, deletion mutants were produced. It was shown that the N-terminal part is responsible for binding of the secondary cell wall polymer (SCWP) and that the C-terminal part is essential for self-assembly [1].

We present here the first structure of a bacterial S-layer protein. The C-terminally truncated form $\mathrm{rSbsC}_{(31-844)}$ was crystallized [2] and the structure was solved by MIRAS to $2.9 \AA$. It revealed a novel ring-like architecture with an interesting fold at the $\mathrm{N}$-terminus and disordered second part of the molecule. Since the structure was difficult to refine the approximate domain boundaries were determined and new truncation mutants designed. The structure of another truncation mutant was solved to $2.4 \AA$ using the well defined domains from $\mathrm{rSbsC}_{(31-844)}$ structure as a starting model. The structures were compared and flexibility between the domains could be observed. In order to investigate the behaviour of the truncated mutants in solution, SAXS experiments were performed. Surprisingly, the protein behaves differently in the solution showing an elongated conformation. This finding confirmed the large inter-domain flexibility of the protein.
Furthermore, the thermostabiliy of truncation mutants was investigated in presence and absence of SCWP. A drastic stabilization of the protein was observed when SCWP is bound.

[1] Jarosch M., Egelseer E.M., Mattanovich D., Sleytr U.B., Sara M., Microbiology, 2001, 147, 1353.

[2] Pavkov T, Egelseer E.M, Sara M., Keller W., Acta Cryst. 2003, D59, 1466

\section{MS19 P15}

Predicted and experimental crystal structures of nitroanilines and nitrophenols Grażyna Wójcik and Izabela Mossakowska, Institute of Physical \& Theoretical Chemistry, Wroctaw University of Technology, 50-370 Wroctaw, Poland.

E-mail : grazyna.m.wojcik@pwr.wroc.pl

Keywords: crystal structure prediction, polymorphism, nitrobenzenes

The crystal structure prediction experiment has been performed for several single-substituted nitrobenzenes, i.e. ortho-, meta and para-isomers of nitro-, halogeno-, nitrilo-, isonitrilo- and methyl derivatives, as well as nitrophenols, nitroanilines, nitrobezaldehydes and nitrobenzoic acids. The prediction was carried out using the Polymorph Predictor, module of Cerius $^{2}$ program [1, 2]. The Monte Carlo simulated annealing was used for searching crystal structures corresponding to global minimum in the lattice energy for several chosen space groups [3]. An intermolecular force field was used to calculate intermolecular energy, which consisted of coulombic, polarisation, dispersion and repulsion terms [1]. The lattice energy was calculated as a sum of interactions using the atom-atom potential approach. The molecular geometries were minimized using three different methods: a force-field or semiempirical quantum methods, or $a b$ initio RHF/6-31g ${ }^{* *}$.

The results of the prediction procedure concerning dinitrobenzenes, halogenonitrobenzenes, nitrilo- and isonitrilobenzenes, and methylnitrobenzenes were satisfying. The structures predicted successfully (i.e. very similar to the known experimental crystal structures) were found among ten predicted structures of lowest energies. The results of prediction of anilines, phenols, aldehydes and acids were far worse. The crystal structures of 3 isomers of nitroanilines have not been found. The reason for that may consist in an improperly determined charge distribution in those highly polarizable molecules. In case of nitrophenols only the low-temperature polymorphs of $\mathrm{m}$ - and p-nitrophenol have been found. The negligence of temperature and entropic effects may be the reason for that. The crystal structure of o-nitrophenol have not been found. However, the density of the real crystal of onitrophenol is far smaller than the densities of the predicted crystal structures of lowest energies. This result suggests that the known crystal structure may correspond to a high-temperature polymorph. Search for another polymorph is at the moment in progress. The predicted crystal structures will be presented and discussed in terms of important interactions and possible molecular synthons in the crystals.

[1] Leusen, F. J. J., J. Cryst. Growth, 1996, 166, 900

[2] Cerius $^{2}$ Molecular Simulations Inc., San Diego, CA, 1997

[3] Gdanitz R. J. Chem Phys. Letters, 1992, 190, 391 